\title{
Investigation of tensile strength and dimensional variation of T700 carbon fibres using an improved experimental-setup
}

\author{
This is the peer reviewed version of the \\ following article: \\ Journal of Reinforced Plastics and \\ Composites 39(3-4):144-162 \\ CThe Author(s) 2019 \\ Reprints and permission: \\ sagepub.co.uk/journalsPermissions.nav \\ DOI: $10.1177 / 0731684419873712$ \\ www.sagepub.com/
}

\section{Faisal Islam ${ }^{1}$, Sébastien Joannès ${ }^{1}$, Steve Bucknell ${ }^{2}$, Yann Leray $^{2}$, Anthony Bunsell ${ }^{1}$ and Lucien Laiarinandrasana ${ }^{1}$}

\begin{abstract}
Knowledge of fibre strength is crucial for understanding the failure behaviour of fibre reinforced composite materials and structures. Measuring the properties of technical fibres has been known to be very challenging and the different challenges associated with single fibre characterisation are illustrated in this article. An improved and automated experimental methodology for tensile testing of single fibres is described. This process has been used to generate fibre strength data for T700 carbon fibres at three different gauge lengths of 4,20 and $30 \mathrm{~mm}$. The variability in strength and modulus of short fibres was found to be much larger than that of longer fibres. Statistical analysis of this large data set has also highlighted the limitations of the standard Weibull distribution for representing fibre strength behaviour. The need for a better statistical representation of the fibre strength data in order to provide a more accurate description of the fibre strength behaviour has been emphasized.
\end{abstract}

\section{Keywords}

Single fibre testing, Automation, Instrumentation, Composite materials, Dimensional analysis 


\section{Introduction}

Fibres are known to be an essential constituent of many living things. They are present in nanostructures such as twisted strands of DNA and also in large and complex structures such as the muscles and tissues of both mammals and trees. Humankind has taken inspiration from such structures to use fibres for the development of textile materials and fibre-reinforced composites. Fibres are widely used in composite materials to make light-weight and high-strength products. The reinforcement of polymeric matrices by fibres is found to bring about significant advancements in the mechanical behaviour of polymers. This provides added advantages suchg as high strength to weight ratio, excellent weathering stabilities and enhanced dimensional stabilities. This combination can generate some of the strongest and versatile materials that have ever been known or developed.

The outstanding characteristics of composite materials are imparted by the fibres which are used to reinforce the matrix, as these fibres are the principal load bearing constituents of the composite material. Failure of composite materials typically occurs due to the accumulation of fibre breaks. Knowledge of fibre strength is therefore crucial for understanding the failure behaviour of fibre reinforced composite materials and structures. Using computational strength models, which uses the fibre properties as input, a simulation of the effective properties of the composite materials can be made enabling an estimation of failure onset. These models make predictions on the strength and damage behaviour of the composite materials and structures. The predictions are primarily based on the input properties of its constituents amongst other interactions ${ }^{1-3}$. It is therefore very crucial to characterise the fibres accurately to enable the models to make accurate predictions ${ }^{4,5}$. Fibres do not share the properties with the same material in bulk form, whilst some fibres do not even exist in bulk form. Most fibre characterising methodologies encounter numerous difficulties which make the measurement of fibre properties very challenging. Previous work of the authors has shown that there may be significant inaccuracies in experimentally generated fibre strength data ${ }^{6}$. Some of the difficulties associated with fibre strength characterisation are discussed in this article. Advanced experimental techniques if used appropriately can be highly advantageous in overcoming the existing challenges and practical limitations.

Fibre strength is typically represented using a statistical distribution. For an accurate statistical representation of the fibre strength behaviour, a large sample set of fibre strength data is required. The need for a large fibre strength data set for reliable analysis has been highlighted by a number of studies ${ }^{7-10}$. Obtaining a large sample set of fibre strength data can be problematic since the fibre testing process is usually very challenging and time consuming. Most studies on fibre strength characterisation

${ }^{1}$ MINES ParisTech, PSL University, Centre des Matériaux (CMAT), CNRS UMR 7633, BP 8791003

Evry Cedex, France

${ }^{2}$ Dia-Stron Ltd., Andover, United Kingdom

Corresponding author:

Sébastien Joannès

Email: sebastien.joannes@mines-paristech.fr 
have therefore been limited to a small sample size for analysis. Based on the results of this analysis, conclusions are drawn on the strength behaviour of the whole fibre population. It is comprehensible that analysis conducted on a large sample size would provide a more reliable result than those obtained from smaller sample sizes. Therefore, the choice of a sample size for analysis introduces uncertainty nto the results. Any uncertainty in the input fibre strength would also propagate into the composite models. If there is any uncertainty in the accuracy of the fibre strength information, the predictions made by the models on the material and structural behaviour would also be uncertain and unreliable. To reduce this uncertainty, a large and accurate data set of fibre strength is required. The present study addresses these needs.

The objective of this article is to first understand the different limitations associated with fibre strength characterisation. This knowledge would then be used to determine methods which could be followed to improve the fibre characterisation process. These methods would then be implemented to generate and analyse fibre strength data that could be used to determine the representative statistical variations in fibre strength. This has been illustrated by using T700 carbon fibres, a commonly used reinforcement for composite materials in critical structural applications. The study has been conducted at different gauge lengths to compare the results.

\section{Fibre strength characterisation}

Characterising fibres is challenging, as the fibre diameter can be just a few microns especially for brittle technical fibres such as carbon. Despite considerable progress in characterisation techniques, many obstacles still remain to obtain accurate fibre strength data. Fibre strengths cannot be represented by a single average value. They have a probabilistic nature and vary greatly between fibres. Variation in fibre strengths are mostly represented using appropriate statistical distribution functions of which the Weibull distribution is the most popular ${ }^{11}$. To appropriately represent fibre strength, complete information about its tensile strength statistical distribution and the corresponding distribution parameters are required.

There are different methods to obtain fibre strength and its distributions such as the fibre bundle tests, fibre fragmentation tests, single fibre tensile tests, loop tests, etc. However, each method has its own limitations. In the fibre bundle method, a bundle of fibre is tested by applying a load and observing the behaviour of the bundle as a whole. The single fibre fragmentation test embeds a fibre inside matrix and the composite is tested to determine the parameters for the fibre strength distribution. The single fibre test is one of the most straightforward techniques however, it is a very laborious and time consuming process. The next subsections discuss these popularly used techniques for the determination of fibre strength distribution, along with the challenges that are associated with each methodology.

\subsection{Fibre bundle tests}

A bundle containing a large number of fibres is subjected to tensile loading and the load-strain curve is recorded. The load and strain are then represented using an appropriate form of a Weibull distribution and has been discussed previously by a 
number of authors ${ }^{12-14}$. It is assumed that all fibres in the bundle are nominally identical, that they are perfectly aligned and are linear elastic in nature.

Limitations:

- The analysis is based on the assumption of equal load sharing amongst the surviving fibres of identical length. However, the spread of the individual fibre gauge length inside the bundle is unavoidable which may lead to unequal distribution of stress on the fibres. It has been shown that there may be a difference in the bundle length and fibre length. This difference called slack, introduces a non-linearity in the load-strain curve ${ }^{15,16}$.

- The fibres inside a bundle interact laterally with each other. This causes local shearing and contributes to the fibres adjacent to breaks ${ }^{17,18}$.

- When considering load transfer interactions, most strength models for fibrereinforced matrix composites use the idea that the entire surface area of each fibre is wetted by matrix material. Load transfer within the composite is then achieved through each individual fibre-matrix interaction, instead of through the interaction between a group of fibres and the matrix, as in a fibre bundle test.

- Depending upon the type of material and manufacturing process used for making the fibres, there can be significant variations in diameter of different fibres and in some cases, along the length as well. Due to this non-uniformity in fibre dimensions, the bundle test may not be a very appropriate method to determine fibre strength distribution.

\subsection{Fragmentation tests}

In this method, a single fibre is embedded in a matrix and the composite is subjected to a longitudinal incremental displacement. The fibre inside the matrix breaks repeatedly at different locations along the length of the fibre. Polarized light is used to identify the sites of fibre breaks. Ultimately, a saturation point is reached when there are no further fibre breaks and there are a very large number of very short fibre segments. The number of fibre breaks is inspected continuously and is deduced to be a function of the applied load, until the onset of saturation. At each break, the corresponding stress is recorded, and the average fragment length is calculated. The method to obtain the Weibull shape and scale parameter using this process has been described by different authors ${ }^{19-22}$.

Limitations:

- If the bonding at the interface of fibre and matrix is too strong, the fibre cracks may propagate into the matrix and cause the matrix to deform around the crack. On the other hand, if the bonding at the interface is weak, debonding between thematrix and fibre may occur which may result in the fibre slipping out of the matrix. This may change the gap between broken fibres and hamper load transfer to the remaining parts of the fibre ${ }^{23}$.

- The theory of fragmentation requires that the fragmentation data is strictly lognormally distributed. It has also been found in different studies that the fibre 
fragment length distribution indeed follows a log-normal distribution, but only in the early fragmentation stages ${ }^{19,24}$.

- The calculation of fibre stress can be affected by residual stresses developed due to different rates of thermal expansions in fibres and matrix. It has been shown that the Weibull parameters can be very sensitive to residual stresses ${ }^{22,25}$. For calibrating the fibre stresses, residual stresses need to be determined by comparing the fibre stress calculated using fragmentation tests to the stresses calculated from single fibre tests. Alternatively, it can be estimated using a method proposed by $\mathrm{Tsai}^{26}$.

- It is assumed that fibre breaks are non-interacting in nature. This assumption is only valid if the fibre break density is very low. Several models have been developed to capture the effect of fibre break interactions ${ }^{20,27-29}$, some of which add a parameter called exclusion zone length ${ }^{30}$. However, such models are very sensitive to the stress transfer between matrix and fibres requiring very accurate information about the exclusion zone length; determining which is complex and it may also vary with fibre stress.

\subsection{Single fibre tests}

For determining the strength of fibres, individual fibres are tested one by one. In its simplest form, a single fibre is subjected to an increasing tensile load until failure. The measured failure load and fibre cross-sectional area are used to calculate the fibre strength. The test is repeated with a sufficient number of fibres to generate a set of fibre strength data. The data set is then fitted to a representative statistical distribution function, of which the Weibull distribution is the most commonly used. The standard 2-parameter Weibull distribution is given by Equation 1 and a more generalized 3parameter Weibull distribution is given by Equation $2^{11}$. The limitations of using these functions for representing the fibre strength behaviour will be discussed in Section 4 .

$$
\begin{gathered}
P_{\mathrm{R}}(\sigma)=1-\exp \left(-\left(\frac{L}{L_{0}}\right)\left(\frac{\sigma}{\sigma_{0}}\right)^{m}\right) \\
P_{\mathrm{R}}(\sigma)=1-\exp \left(-\left(\frac{L}{L_{0}}\right)\left(\frac{\sigma-\sigma_{u}}{\sigma_{0}}\right)^{m}\right)
\end{gathered}
$$

Where; $P_{\mathrm{R}}(\sigma)$ is the probability of fibre failure for an applied stress $\sigma ; L$ being the characteristic gauge length; $L_{0}$ the reference gauge length; $\sigma_{0}$ the scale parameter; $\sigma_{u}$ the location parameter and $m$ the shape parameter or Weibull modulus. The expression given by Equation 1 is a special case of the Weibull distribution. It is obtained by starting with the generic 3-parameter Weibull distribution function given by Equation 2 and then fixing the location parameter $\sigma_{u}$ to be 0 .

Many standards are available that describe the methodology for testing single filament materials such as BS ISO $11566^{31}$, ASTM C1557 ${ }^{32}$, ASTM D3379-75 33 , etc. The common steps followed in the single fibre testing process for determining fibre strength are as follows: 
(1) A single fibre is removed from the fibre bundle and mounted on a card or cardboard frame with a central cut-out region ${ }^{6}$, as shown in Figure 1(a).

(2) The ends of the fibre are then glued to the card or paper frame using epoxy, wax, or some other suitable adhesive at locations depending upon the required gauge length.

(3) The diameter of the fibre is usually measured at this moment using an appropriate measuring technique.

(4) The frame is gripped in the jaws of a tensile testing machine at the two ends.

(5) The sides of the card frame are cut which allows any applied load to be transferred directly to the fibres.

(6) The jaws of the testing machine are then pulled until the fibre breaks and the failure load of the fibre is recorded.

(7) The set of fibre strength values are used to find a representative Weibull distribution.
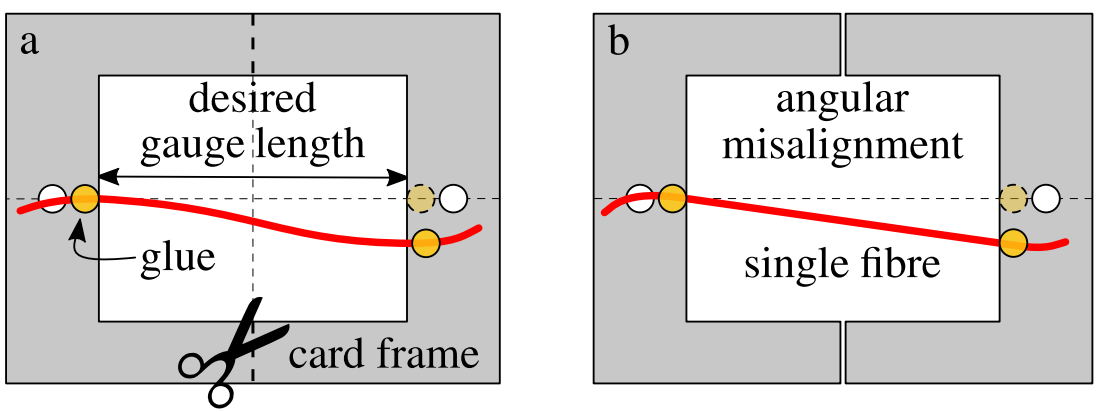

Figure 1. (a) Schematic diagram of a single fibre mounted on a card frame, (b) Angular misalignment in the fibre specimen

The entire process of specimen preparation and testing is very cumbersome and time consuming. One major source of error with this method of specimen preparation is improper fibre alignment, as shown in Figure 1(b). An actual image of a card frame with a single fibre mounted on it is also shown in Figure 2. Since the glue on the right end is not perfectly aligned with the one on the left, it has resulted in a slight misalignment of the fibre. This may also result in inaccurate measurement of gauge length. The quality of the data generated can be improved by addressing the sources of error which would minimise their impact on measured fibre strength and improve the accuracy. A sufficient number of single fibre strength data points are required to ascertain the parameters of the best fit statistical distribution which represents the 


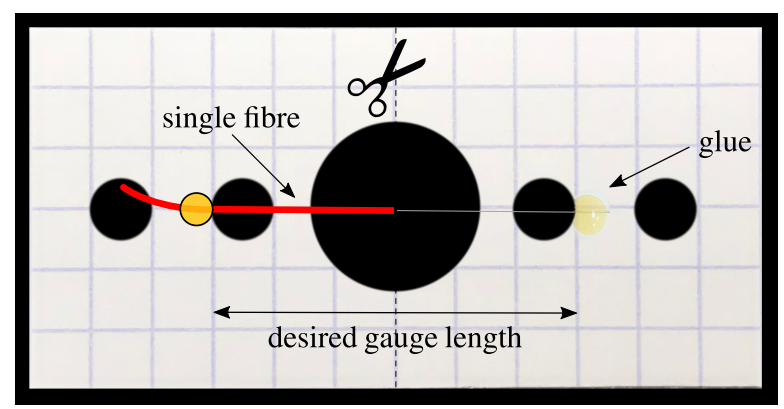

Figure 2. Actual image of a card frame with a single fibre mounted on it

fibre strength variation of the whole population. The time taken to generate a large experimental data set using this manual testing method is very long. Conversely, if a small data set is used then the representativeness of the fibre strength data set would be questionable due to sampling randomness. To generate a large data set, an improved and automated testing setup is required.

\section{Limitations:}

- Single fibre testing is a labour intensive and time consuming process.

- Accuracy of results depend strongly on the sample size used, which requires testing a large number of fibres.

- For proper execution of the test, it is important that the fibres are well aligned in the direction of applied load which depends upon the accuracy of the size and shape of paper tabs and proper mounting of fibres on top of these tabs. Since these steps are usually carried out manually, accuracy of the tests depends strongly on operator skills.

- It is possible that weak fibres may break during the extraction process. Hence, extreme care is required to protect the fibres from any unwanted forces which may break the fibres.

Contemplating the different limitations that each process contains, it seems that the classical single fibre testing process is still one of the most reliable and unambiguous means of characterising fibres or exploring their morphologies. The different individual steps in single fibre testing, however, leave scope for improvement. If the existing problems can be solved, the efficiency of the process can be significantly improved. Several issues concerning mostly the single fibre testing process that require special attention are discussed in section 2.4. 


\subsection{Critical issues for determining fibre strengths}

Some important issues that influence the process of single fibre testing or affect the determination of the fibre strength distribution are listed as follows:

2.4.1 Proper alignment. It is important to ensure that the applied load is properly transferred to the fibre during a test. A small degree of fibre misalignment may lead to an incomplete load transfer. This may result in improper measurement of the failure $\operatorname{load}^{34}$ and incorrect calculation of fibre strength. Bending stresses may also develop at the ends of fibres which may lead to failure of fibres at the clamping/bonded location $^{35-37}$.

2.4.2 Number of tests. Thomason ${ }^{7}$ and Berger et al. ${ }^{8}$ have studied the effect of sample size on the estimated fibre strength distribution. It has been shown that using a small number of fibre strength results for the analysis may cause wrong conclusions about the behaviour of strength distribution and related fibre properties. However, there is no general agreement amongst the different studies on the number of tests required to fully describe the strength behaviour of a fibre population ${ }^{38-40}$. Therefore, to avoid misinterpretation, a sufficient number of fibre strength data points are required. The number of experimental repeats should be decided accordingly depending on the testing method chosen. The accuracy of a statistical function that is used to represent the fibre strength distribution could be improved substantially if a large number of fibre strength data points are available for analysis. This would increase the reliability of the calculated parameter values for the representative statistical distribution. Assigning a level of confidence to fibre strengths and distributions using statistical techniques can be another way to add meaningful inferences about the population behaviour ${ }^{6}$. This also depends on the size of the fibre strength data set used for analysis.

2.4.3 Fibre extraction. Since the diameters of fibres are very small, the break force is very low. Many weak fibres therefore may break during the process of fibre extraction from a bundle and specimen preparation ${ }^{41,42}$. As a result, the weaker portion of the fibre strength distribution may not be represented in the experimentally generated fibre strength data set. The elimination of weak fibre strengths would result in an incomplete data set which does not represent the entire fibre population correctly ${ }^{12,43}$.

2.4.4 Accurate measurement of cross sectional area. For accurate calculation of fibre strength, it is vital that the applied load is calculated properly and the exact cross sectional area of the fibre is accurately determined ${ }^{44-47}$. Any error measured in cross sectional area will affect the accuracy of the calculated fibre strength ${ }^{48}$.

2.4.5 Optimum gauge length. Composite material strength models require input fibre strength information at very small gauge lengths, usually in the range of micrometres to a few milimetres ${ }^{10}$. Testing fibres at such small gauge lengths is practically impossible. A method of extrapolation is therefore used to determine strengths at very small gauge length fibres from strengths of long fibres obtained experimentally. Testing at either very short or very long gauge lengths is associated with respective complications. It is therefore important to determine an appropriate gauge length that is optimum for testing ${ }^{36,37}$. 
In the following sections, an improved and automated procedure for testing single fibres will be discussed which can help in overcoming the problems faced by the standard single fibre testing method.

\section{Material and methods}

\subsection{Material}

T700 carbon fibres are very popularly used in fibre reinforced composites for structural applications. Bobbins of T700SC carbon fibres manufactured by Toray were used for the present study. The properties provided by the manufacturers data sheet are given in Table 1. Fibre dimensional analysis and single fibre tensile tests were conducted on the fibres extracted from the procured bobbins.

Table 1. Fibre properties supplied by the manufacturer

\begin{tabular}{lccc}
\hline Characteristics & Unit & $\min$ & $\max$ \\
\hline Tensile Strength & $\mathrm{MPa}$ & 4510 & - \\
Young's Modulus & $\mathrm{GPa}$ & 221 & 240 \\
Elongation at break & $\%$ & 1.9 & - \\
\hline
\end{tabular}

\subsection{Methods}

3.2.1 Fibre dimensional measurement method. The previous work of the authors has highlighted the importance of dimensional measurement of fibres for accurate estimation of fibre strength ${ }^{6}$. It has been statistically established that fibre diameter is the most critical parameter for accurate determination of fibre strength. To determine the variations in fibre diameter and cross-sectional area, an automated and non-contact measurement system, the Fibre Dimensional Analysis System (FDAS770) was used. The FDAS770 is based on the principles of laser scanning microscopy and installs a Mitutoyo laser scanning micrometre (LSM500). The system allows non-contact, rapid and accurate fibre dimensional measurements and has also previously been used for studies involving dimensional analysis of different types of fibres ${ }^{49}$. An isometric view of the setup is shown in Figure 3(a), its working in Figure 3(b) and a zoomed-in image of the translation stage is shown in Figure 3(c).

To ensure that the fibres do not fail during the process of handling and transfer to the measurement setup, the specimens were handled using a fixture specifically designed to lift and manoeuvre the fibres. This fixture also ensured that the fibre gauge length is fixed and that there is no tension on the fibre specimens. After placing the fibre specimen on the setup, the rotating sample arms allow a complete 360-degree inspection of the fibre. The linear translation stage allows different fibre segments to be measured in the axial direction, i.e. along the length. The combined rotation and translation movements provide a complete 3-dimensional analysis of the fibre. 
The system facilitates a measuring range of 5-2000 micrometres with an accuracy of \pm 0.3 micrometres.

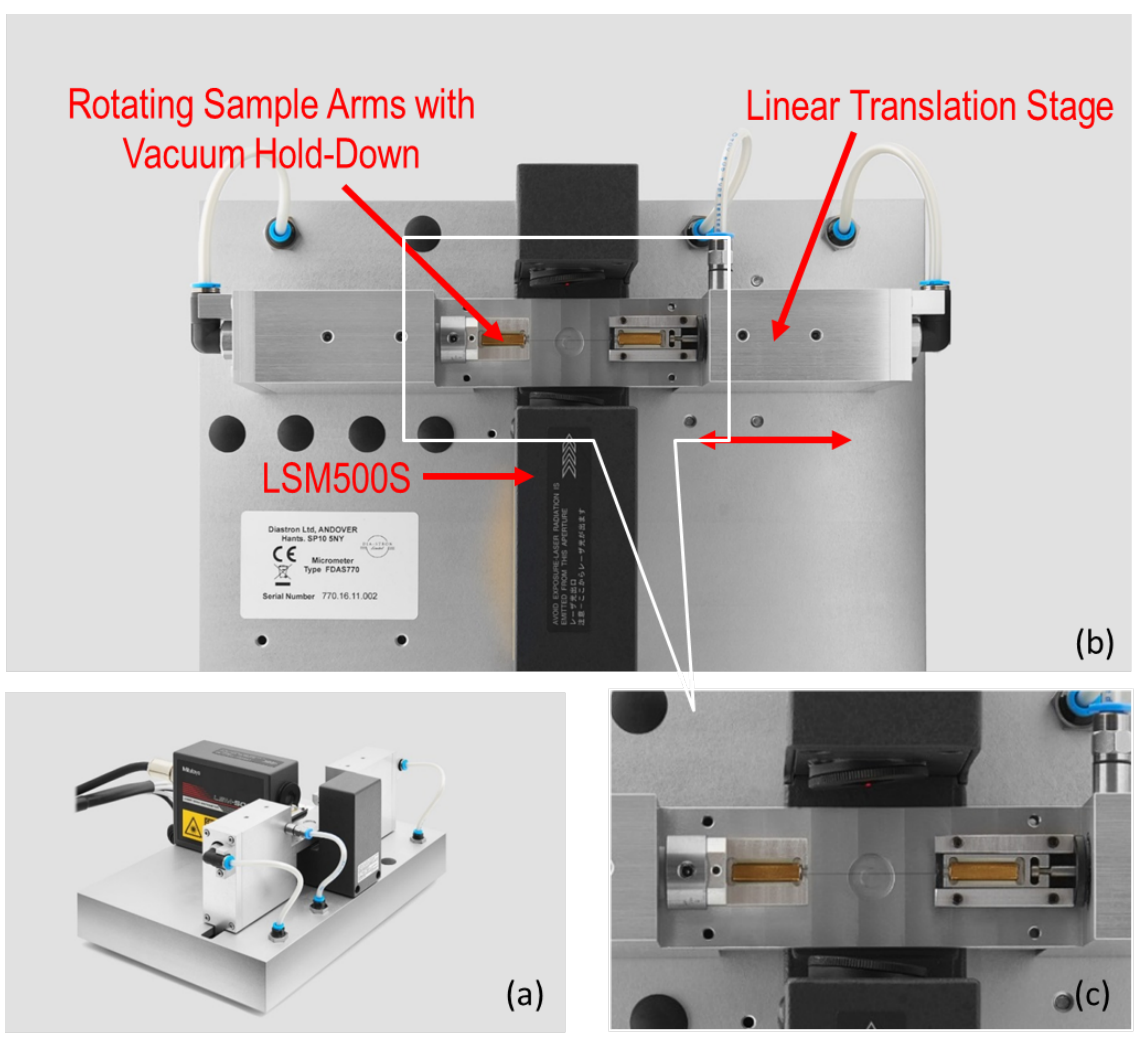

Figure 3. (a) Dia-Stron Fibre Dimensional Analysis System (FDAS770, Isometric view). (b) Working of the FDAS770 (top view). (c) Zoomed-in image of the translation stage.

3.2.2 Improved single fibre testing method. To overcome the challenges faced by the conventional method of testing single fibres, an improved single fibre testing setup has been developed. This experimental setup can be used to generate accurate single fibre strength data for different types of fibres. The entire testing process for determining the strength of a single fibre has been divided into 3 stages. Improvements introduced in each stage to address the existing challenges will be discussed in the following subsections. The stages include:

- Specimen preparation

- Specimen loading

- Specimen testing 
Specimen preparation. The use of paper frames has been replaced by plastic tabs, which are shown in Figure 4(a). These tabs impart rigidity to the prepared specimen. Single carbon fibres are randomly selected from the fibre yarn, and each end of the fibre is mounted on a plastic tab. The tabs are separated by a distance equal to the required gauge length, which is defined by exclusive cassettes which also act as a platform for holding all prepared specimens. A fibre selection and vacuum pick-up pen assists with filament separation from the yarn. The plastic tabs have fine grooves at the ends which prevents any lateral movement of fibres on the tab. For this, each end of a fibre is supported on 2 grooves of a tab (the other 2 grooves are disregarded). The wells at the fibre ends (one at each end) are then filled with an adhesive which then locks the fibre in its position. This ensures excellent alignment and also fixes the gauge length of the fibre specimen very accurately, as also shown in Figure 4(b). However, while using the classical method of sample preparation using a paper tab, the misalignment is much larger (few millimetres). Using the plastic tab thus brings significant improvements in specimen preparation and in minimising misalignment. EMI Optocast $3553 \mathrm{UV}$ curing adhesive was used to bond the fibres to the tabs. The adhesive on each tab was cured by exposing it to UV light at a wavelength of $365 \mathrm{~nm}$ for about 15 seconds. Figure 5 shows a cassette loaded with 20 fibre specimens. Each specimen comprises of two tabs holding a single fibre in its grooves.
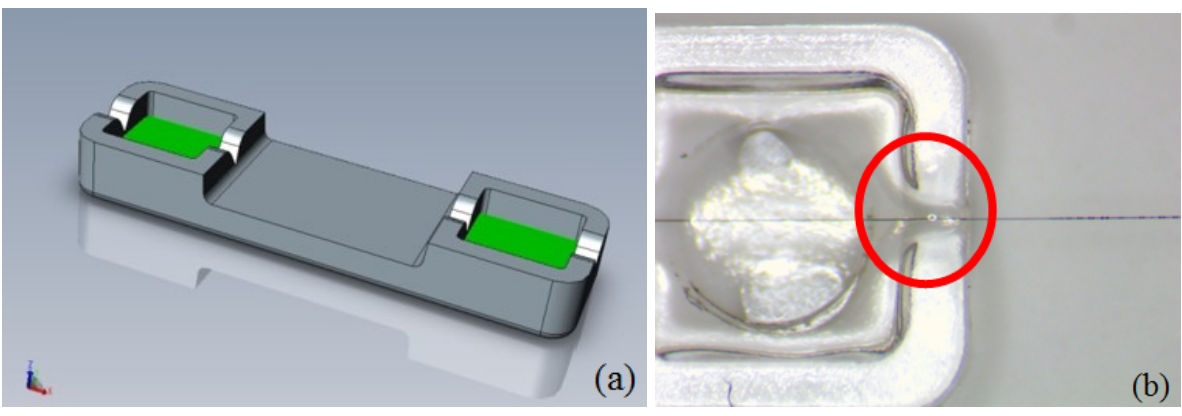

Figure 4. (a) Plastic tabs used for mounting one end of a fibre. (b) Well defined gauge length with proper alignment.

Specimen loading. Once the cassette was ready with a set of fibre specimens, each specimen was transferred from the cassette to the testing site via an Automated Loading System (ALS1500), shown in Figure 6. The system operates automatically using vacuum suction which allows efficient and safe transport of the fragile specimens from the storage cassette to the measurement modules. The main benefits of using the automated loading system are: (1) increase in the testing productivity, due to a continuous and unsupervised process, (2) reduction in the number of specimens that fail during transport, due to the elimination of most manual handling errors during specimen transfer and mounting, and (3) improvement in data quality, due to the avoidance of unwanted and inconsistent handling loads. 


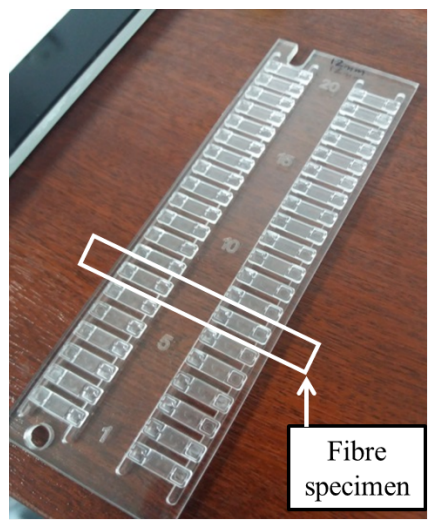

Figure 5. A 20 sample cassette loaded with fibre specimens ready to be tested.

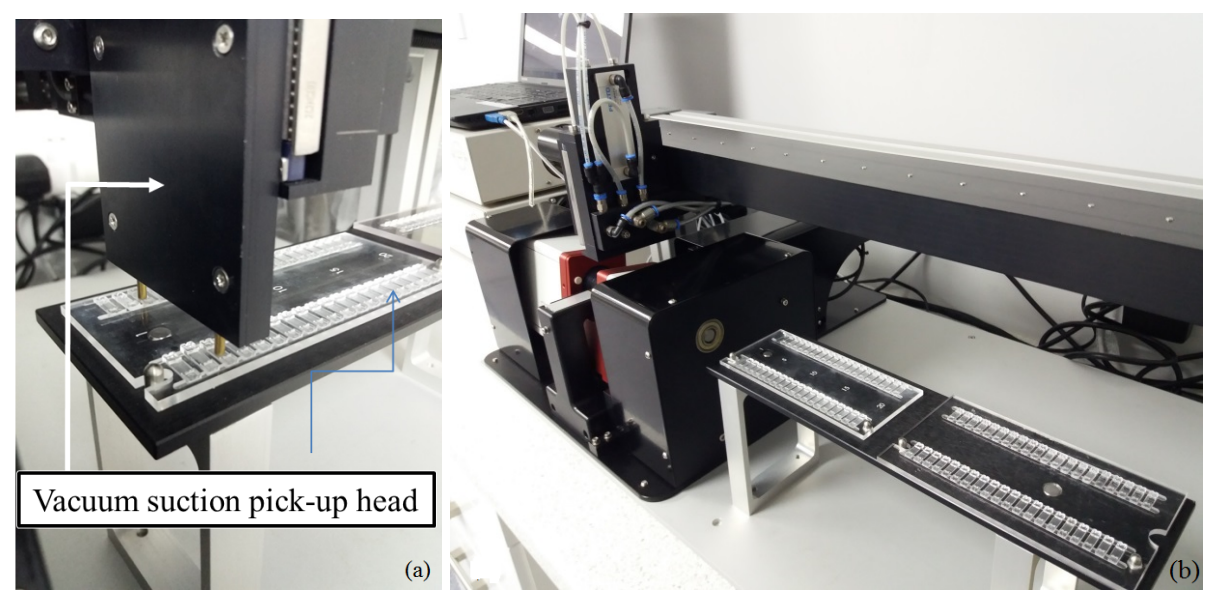

Figure 6. (a) ALS Vacuum suction for specimen pick up (b) transfer of specimen to testing site.

Specimen testing. After collecting and loading a sample, the mechanical testing is conducted by an automated system called LEX/LDS. This system integrates a Linear Extensometer (LEX820) with a Laser Diffraction System (LDS0200) into one module, and is shown in Figure 7(a). The whole setup around the testing site is shown in Figure 7(b). The LEX820 is a high resolution extensometer developed specifically for fine fibre applications. A DC micrometer drive offers exceptionally smooth travel combined with high positional repeatability. The module is useful for fibres which fail at low strain values and provides highly detailed stress/strain data. This makes it very suitable to test brittle technical fibres such as carbon. The LEX820 ensures fibre straightness and orthogonality with the laser beam for high precision diameter 
measurements. It also offers a high range of extension up to $53 \mathrm{~mm}$, and a high range of extension speed up to $2.6 \mathrm{~mm} / \mathrm{s}$.

The LDS0200 provides direct, non-contact diameter measurements of the fibres before tensile tests. The measurement principle is based on laser diffraction which enables diameter measurements down to a few microns. For the calculation of tensile strength, fibre cross sectional area is calculated using the measured diameter. The technical specifications and compliance correction for conducting the tests were in conformance with the standard ASTM C1557-14.
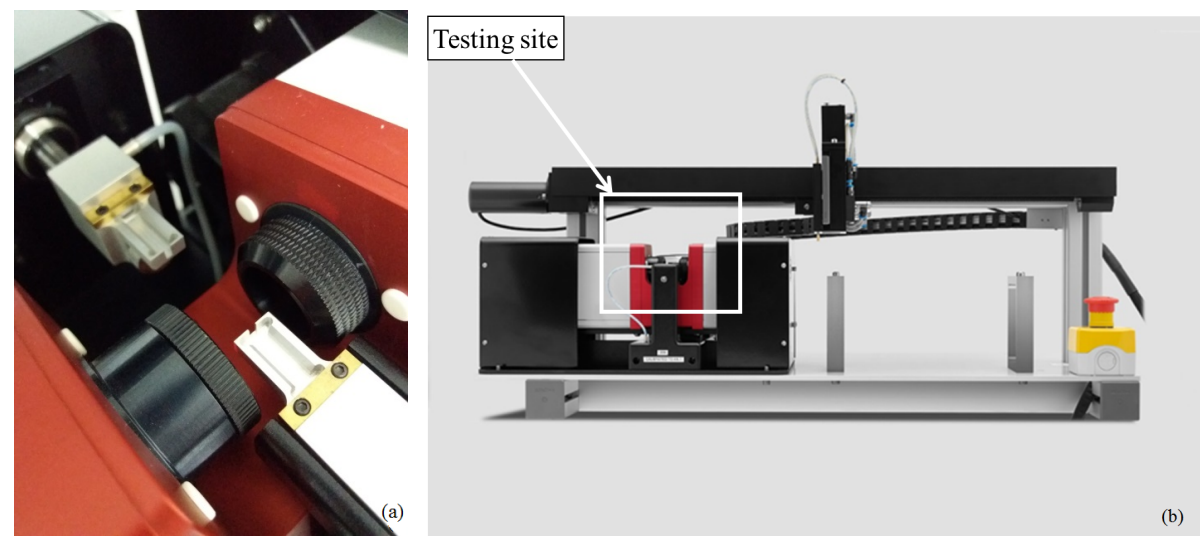

Figure 7. (a) Testing site: Combination of the LEX/LDS system. (b) Testing setup installed with the LEX/LDS system ${ }^{50}$.

\subsection{Experimentation}

3.3.1 Fibre dimensional measurement. A detailed dimensional study on T700 carbon fibres was conducted to capture any dimensional irregularities in such fibres. This was in addition to the fibre diameter measured for calculating the tensile strength of fibres. A total of 10 fibres were used for this study, the length of each fibre being $25 \mathrm{~mm}$. Fibre specimens were measured using the FDAS770 system to measure the apparent diameter of the fibres. Fibre dimensional measurements were taken by first fixing T700 carbon fibres centrally onto the stage and measuring the apparent fibre diameter. Variation in fibre diameter along the length was captured by shifting the stage linearly, i.e. along the axial direction of fibre. The measurement was taken along the total length of $25 \mathrm{~mm}$ at intervals of $1 \mathrm{~mm}$. To measure the fibre diameter variation in the angular direction of the fibre (or due to angular orientation), the fibre specimen was rotated by 10 degrees about its axis. The fibre diameter was again measured similarly along the entire length, for this new angular position. This was repeated for 19 different angular positions from 0 up to 180 degrees, at equal intervals of 10 degrees. The combined rotation and translation of the fibre together provided dimension measurements at a total of 475 , i.e. $25 \times 19$, different locations on the fibre surface. This has been schematically represented in Figure 8 by unwrapping the 
fibre surface on a 2-D plane. The different central points at which fibre dimensions were recorded are shown by the intersection of the horizontal and vertical lines in Figure 8(c). The results are discussed in section 4.1.
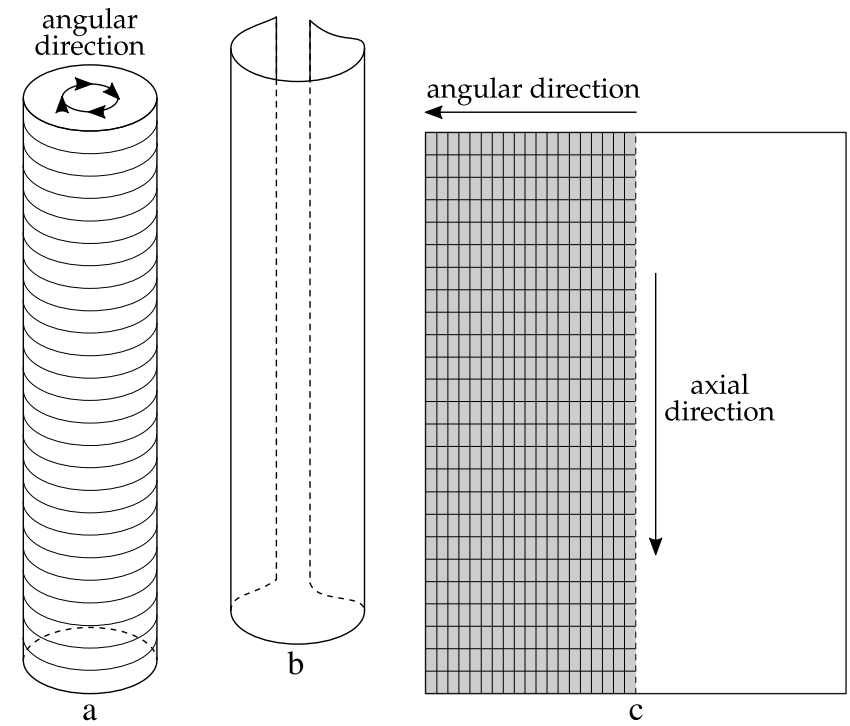

Figure 8. Schematic diagram of unwrapping a fibre surface on a 2-D plane to show the different locations at which dimensional measurements were recorded.

3.3.2 Fibre tensile strength measurement. T700 carbon fibres were tested for tensile strength using the automated testing system described in section 3.2.2. Fibres were tested at three different gauge lengths of $4 \mathrm{~mm}, 20 \mathrm{~mm}$ and $30 \mathrm{~mm}$. A requirement for composite strength models is that the input fibre properties should be defined at specific gauge lengths. These required lengths are usually smaller when compared to the gauge length usually used for experimentation, mainly because practical limitations make it difficult to conduct experiments at very short gauge lengths. For most models, fibre properties for gauge lengths in the range of a few hundred micrometres to a few millimetres are required. ${ }^{1,3,51}$ However, almost all experimental results available are for fibre gauge lengths in the range of a few tens of milimetres ${ }^{10}$. The usual practice is thus to use results generated using long gauge lengths and then to extrapolate the properties to shorter gauge lengths using appropriate statistical methods and models. This has also been the idea behind choosing the gauge lengths for the tests. $30 \mathrm{~mm}$ and $20 \mathrm{~mm}$ are the most commonly used lengths while the composite strength model requires input properties for fibres of $4 \mathrm{~mm}$ gauge length. Testing fibres at three different gauge lengths also allowed the compliance of the system to be calculated, which was then used to calibrate all the measurements, as discussed in the later sections. The automated system allowed testing a large sample set of fibres in a relatively short time 
period. The total number of fibre strength results determined for each gauge length are given in Table 2 . This number is much larger than any of the previously reported studies.

Table 2. Total number of fibre strength results determined for each gauge length

\begin{tabular}{ccc}
\hline S.N. & $\begin{array}{c}\text { Gauge Length } \\
(\mathrm{mm})\end{array}$ & $\mathrm{N}$ \\
\hline 1 & 4 & 120 \\
2 & 20 & 135 \\
3 & 30 & 350 \\
\hline
\end{tabular}

\section{Results and discussion}

\subsection{Fibre dimensional analysis}

The variations in measured fibre diameters are represented with histograms for each gauge length, as shown in Figure 9. The range and frequencies of the fibre diameters were found to be very similar for all the three gauge lengths, as given in Table 3. It can be seen that all three measures of central tendencies, i.e. the mean, median and mode are similar for the three cases.

(a) $30 \mathrm{~mm}$

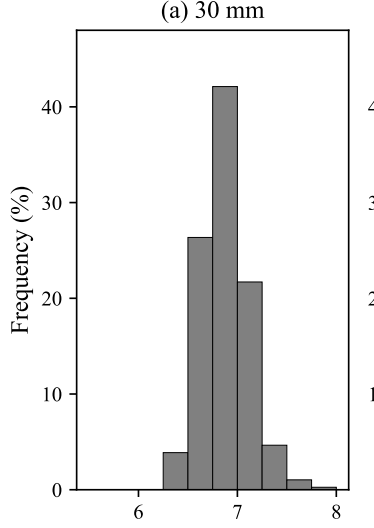

(b) $20 \mathrm{~mm}$

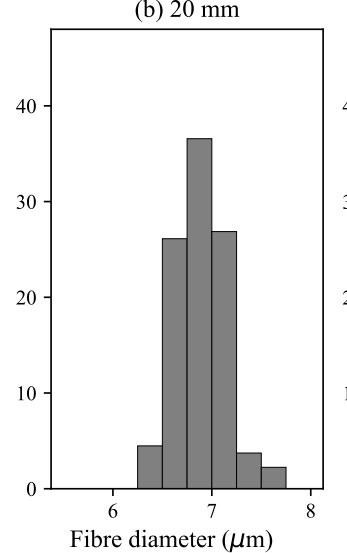

(c) $4 \mathrm{~mm}$

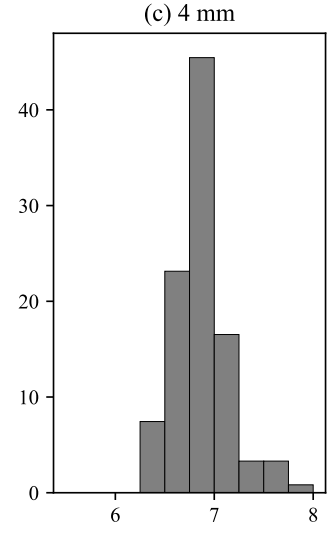

Figure 9. Histograms showing the variations in measured fibre diameters for all fibres of the different gauge lengths: a) $L_{0}=30 \mathrm{~mm}$, b) $L_{0}=20 \mathrm{~mm}$, c) $L_{0}=4 \mathrm{~mm}$

4.1.1 Variation of fibre diameter in axial direction. Figure 10(a) shows the variation in measured fibre diameter along the axis for one of the fibres investigated (say fibre 1). 
Table 3. Mean, median and mode values of fibre diameters for the different gauge lengths

\begin{tabular}{lccc}
\hline Gauge Length $\rightarrow$ & $30 \mathrm{~mm}$ & $20 \mathrm{~mm}$ & $4 \mathrm{~mm}$ \\
\hline Mean $(\mu \mathrm{m})$ & 6.87 & 6.88 & 6.86 \\
Median $(\mu \mathrm{m})$ & 6.86 & 6.89 & 6.83 \\
Mode $(\mu \mathrm{m})$ & $6.75-7.00$ & $6.75-7.00$ & $6.75-7.00$ \\
\hline
\end{tabular}

Different plots represent measurements at different angular orientations. Only results at intervals of $20^{\circ}$ are shown for clarity. The apparent diameter measured was found to be very consistent and varied between $7.4-7.6 \mu \mathrm{m}$ along the length, which is less than $2 \%$ from the mean value. When the measurement was repeated using the same fibre, similar results were obtained with the same locations of maxima and minima, which confirms the reliability of the measurements. Although fibre diameter on average was found to vary in the range $6.8-7.8 \mu \mathrm{m}$ between different fibre specimens, the variation for individual fibres was found to be within 2-3\% along the length for most fibres.

However, for two fibres of those investigated, a much larger variation in apparent fibre diameter was observed along the length, as can be seen in Figure 10(b) (say fibre 2). The apparent fibre diameter in this case is seen to vary in the range 7.2 $8.0 \mu \mathrm{m}$ along the length which is about $10 \%$ of the mean fibre diameter and is a significantly large variation. If such a fibre were used for measuring fibre strength, the exact location where fibre diameter was measured would strongly influence the fibre strength value; the measured fibre strength can then vary by almost $20 \%$ of the mean fibre strength. This may also affect the accuracy of the corresponding estimated Weibull strength distribution.

4.1.2 Variation of fibre diameter in angular direction (orientation). It was also noticed from Figure 10(b) that for a fixed position along the length of fibre, the diameters measured for different angular positions were not constant. This suggests a possibility of non-circularity in fibre cross-section. To examine this, measured fibre diameters at specific locations on the axial axis, and for different angular positions were plotted on a polar scale. Polar plots for fibre 1 and fibre 2 at one particular position are shown in Figure 11. It can be seen from Figure 11(b) that fibre 2 shows variations in diameter in the angular direction, i.e. the apparent cross-section was not circular but more of an elliptical shape. Fibre 1 on the other hand, was found to have a very circular cross-section.

The level of non-circularity may vary from fibre to fibre, but it was observed that the fibres which showed variations in diameter along the length also showed noncircularity in cross-sections. Due to these variations in fibre dimensions, the classical assumption of assuming a circular and constant cross section along the length may not always be appropriate. Measuring the fibre diameter at only one location and orientation in order to determine the fibre stress can result in inaccurate fibre strength calculation. A hypothesis can be made that it might be a better practice to first measure the fibre diameters at different angular positions to determine the cross sectional area 

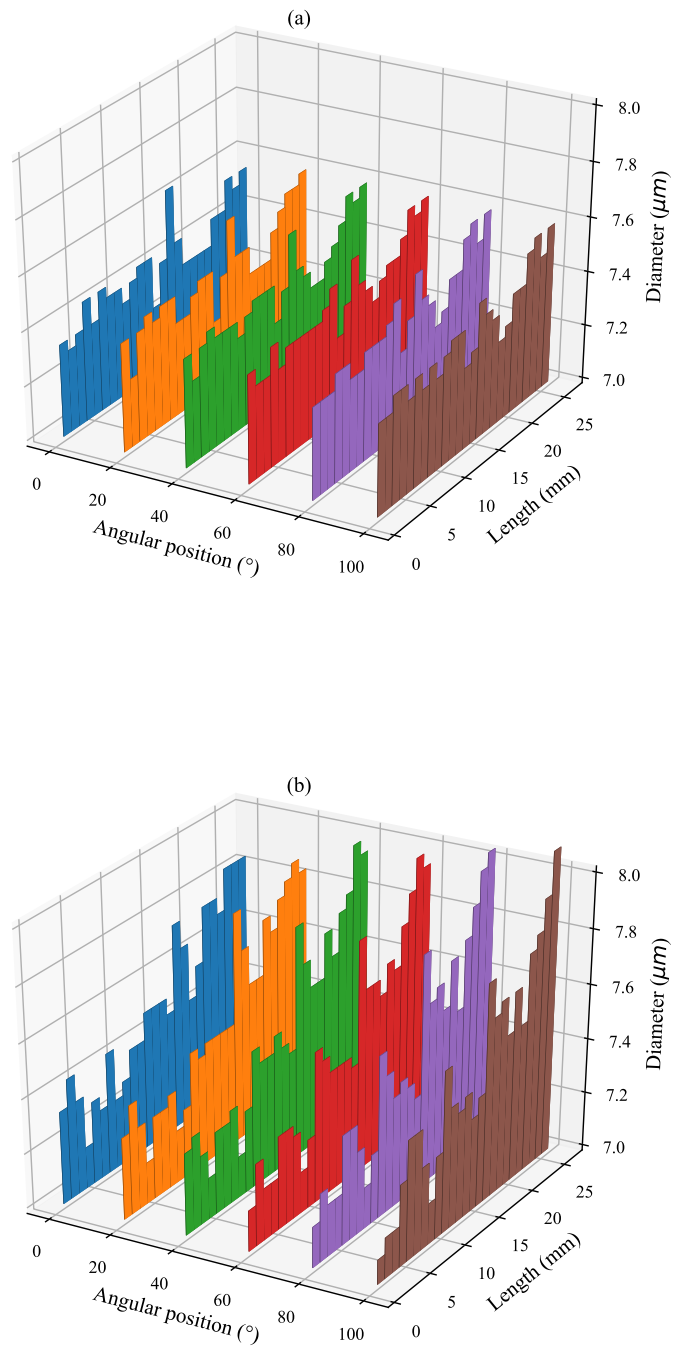

Figure 10. Variation in apparent fibre diameter along the axial length for different angular positions: (a) for fibre 1. (b) for fibre 2

and then additionaly measuring cross sectional areas at different axial positions along the length to cover the entire gauge length. The minimum calculated cross sectional area can then be used for calculating the fibre stress. This may increase the accuracy of the calculated fibre strength. This is because the probability of fibre fracture at the 

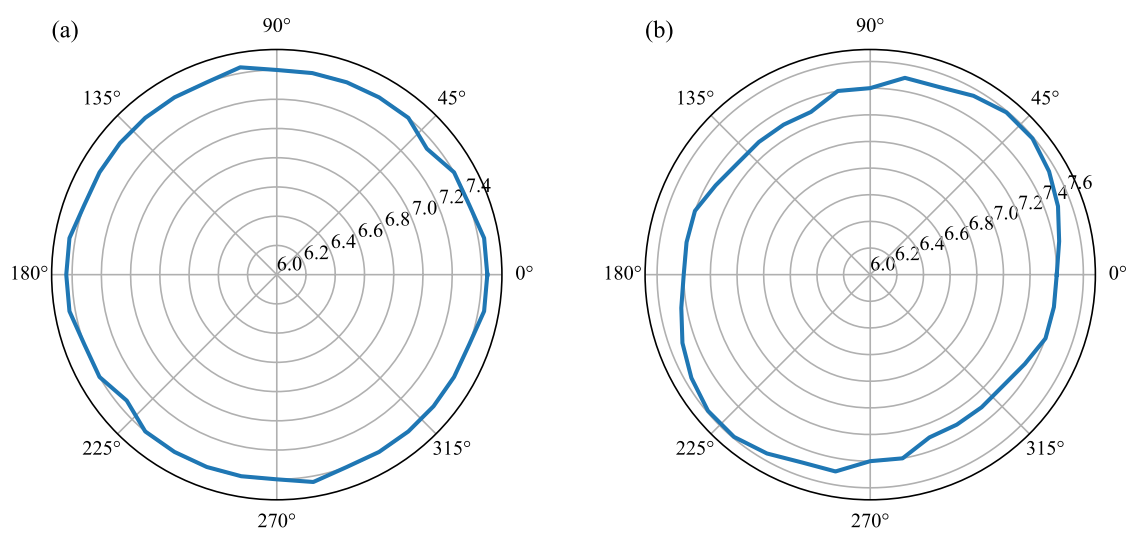

Figure 11. Variation in fibre diameter along the angular direction (apparent cross-section) for: (a) fibre 1 (b) fibre 2. Unit: micrometres.

narrowest region may be higher due to increased stress concentration in that region. This hypothesis would be analysed in the next subsections.

If the non-circularity of fibres is considered, using the standard Weibull functions given by Equations 1 and 2 would no longer be appropriate; due to the variation in fibre cross-sections along the length. The more generalized Weibull functions based on the volume relationship may be more useful in such cases. The term $L / L_{0}$ in Equations 1 and 2 should therefore be replaced by $V / V_{0}$, where $V$ is a characteristic gauge volume and $V_{0}$ is a reference gauge volume.

\subsection{Tensile strength}

The manufacturer's data sheet provides one estimated value for the strength and failure strain of fibres, as given in Table 1. However, these values are rarely found to be the case and usually a variation is observed in strengths of different fibres, as in the present study. The tensile strength for all gauge lengths was found to vary from 2 to $8 \mathrm{GPa}$ with most fibres having a strength between 3-6 GPa as can be seen from the boxplot in Figure 12. Fibres with higher strength values (say, above $6 \mathrm{GPa}$ ) were mostly observed for the specimens with a $4 \mathrm{~mm}$ gauge length, and lower strength values were associated for the fibres with longer gauge lengths of $20 \mathrm{~mm}$ and $30 \mathrm{~mm}$. The solid horizontal line represents the median strength value while the dashed horizontal line represents the mean strength value, for each gauge length. Fibre strength on average was found to decrease with increasing gauge length. This strength behaviour is also in agreement with the Weibull weakest link theory, according to which, fibres of short gauge length are comparatively stronger than fibres of longer gauge lengths. The failure of brittle fibres is controlled by the distribution of defects. For longer gauge lengths (or for larger volumes), the probability to meet a critical defect increases; this increases the probability of failure for longer fibres. 


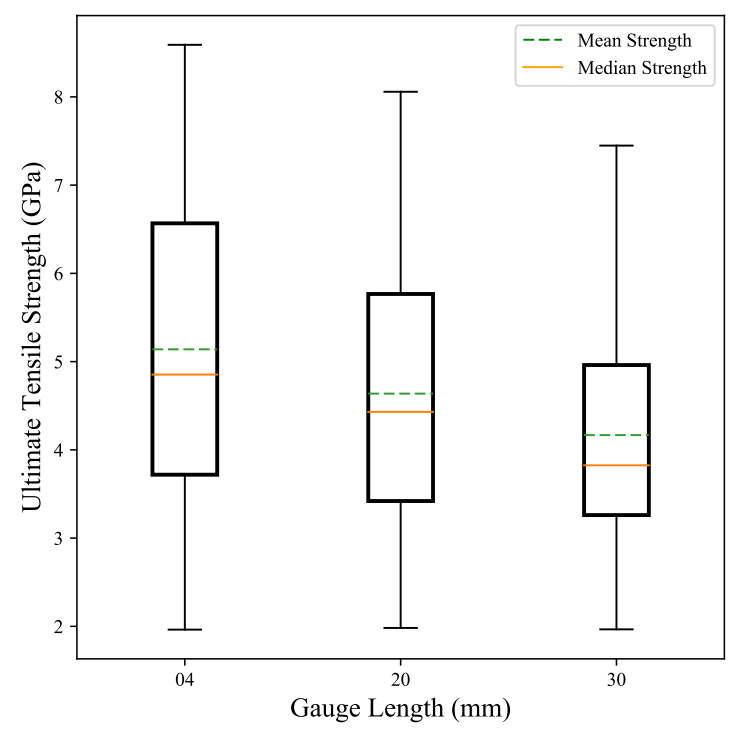

Figure 12. Boxplots for fibre strengths for the three gauge lengths with mean and median values

Strengths of all the fibres were plotted against the corresponding failure strain for all gauge lengths, and are shown in Figures 13, 14 and 15. The histograms for fibre strength and failure strain can be seen to be very similar for all the three cases. The straight line on the plots represents the average Young's modulus of the fibres. The average Young's modulus of the $30 \mathrm{~mm}, 20 \mathrm{~mm}$, and $4 \mathrm{~mm}$ fibres was found to be 232.6 GPa, 231.0 GPa and 234.3 GPa, respectively, as also given in Table 4. This is well within the range prescribed by the manufacturer, which is 221-240 GPa. However, there was an increase in the coefficient of variation in the Young's modulus of fibres with decreasing the gauge length. This is also apparent from the increased scatter in fibre strength data points, especially for tests at gauge length of $4 \mathrm{~mm}$ (Figure 15). This is because of the many experimental issues in conducting tensile tests with fibres of very short gauge lengths. The tensile test measurements at short gauge lengths have earlier been shown to be prone to measurement errors due to an increased effect of fibre misalignment ${ }^{6}$. It should also be emphasized that the Young's modulus was determined between strains of $0.2-1.2 \%$ for all fibres. Since fibres are known to stiffen at higher values of applied strain ${ }^{52}$, the fibres which break at high strain values have slightly higher Young's modulus than the average value, i.e. points at the ends of the strain distribution lie slightly above the straight line of average Young's modulus, as seen in the strength vs strain scatter plots. 
Table 4. Mean, standard deviation and coefficient of variation (CoV) of the average Young's modulus for tests done at the three gauge lengths

\begin{tabular}{lccc}
\hline Gauge Length $\rightarrow$ & $30 \mathrm{~mm}$ & $20 \mathrm{~mm}$ & $4 \mathrm{~mm}$ \\
\hline Mean (GPa) & 232.6 & 231.0 & 234.3 \\
Standard deviation (GPa) & 10.4 & 12.1 & 24.6 \\
CoV (\%) & 4.46 & 5.25 & 10.5 \\
\hline
\end{tabular}

Strengths of fibres were also plotted against their diameters, as shown in Figure 16. The mean diameter value of all the fibres was $6.9 \mu \mathrm{m}$. No specific correlation was observed between fibre strength and their mean diameters. This suggests that fibre strength is not controlled by its size or cross-sectional area but by the distribution of defects inside them. Since critical defects are randomly distributed inside the fibres, irrespective of their cross-section, the fibre strength is also randomly scattered. The hypothesis made earlier that considering the non-circularity of fibre cross-section might result in more accurate fibre strength measurements can therefore not be verified. It has also been shown in a previous study that although fibre diameter measurement is the most critical parameter for accurate fibre strength measurement, minor non-circularity may not affect the fibre strength distribution significantly, and can thus be ignored. ${ }^{5}$

Even if the average fibre tensile strength is within the range reported in the literature, there may be differences in the statistical representation of the experimental results. This is because fibre strengths are typically reported in terms of their best fit Weibull distribution parameters. Even for the same type of fibre, Weibull parameters reported by different authors have been different. This is mainly because of the small number of fibres tested for most studies; thus resulting in uncertainty in the determined properties. ${ }^{53}$

\subsection{Strength distribution}

Due to large differences in individual strength values, strength of fibres cannot be represented using an average value. As mentioned earlier, Weibull distribution is the most popular statistical function used to represent strength of brittle fibres. An advantage of using a logarithmic statistical function such as the Weibull distribution is that it offers a very informative 2-D graphical plot that helps to convey the analysis results visually. The experimental fibre strength data points generated for all three gauge lengths were plotted against their respective cumulative failure probabilities. This has been represented using logarithmic scales as shown in Figure 17. The vertical axis represents the failure probability which is the percentage of fibre population that is expected to fail. The horizontal axis represents corresponding tensile strength. It can be clearly seen that the data points follow a non-linear curvature. The popularly used 2-parameter standard Weibull distribution, which represents a straight line on the logarithmic scale may therefore not be the most appropriate statistical representation for the fibre strength data generated. 

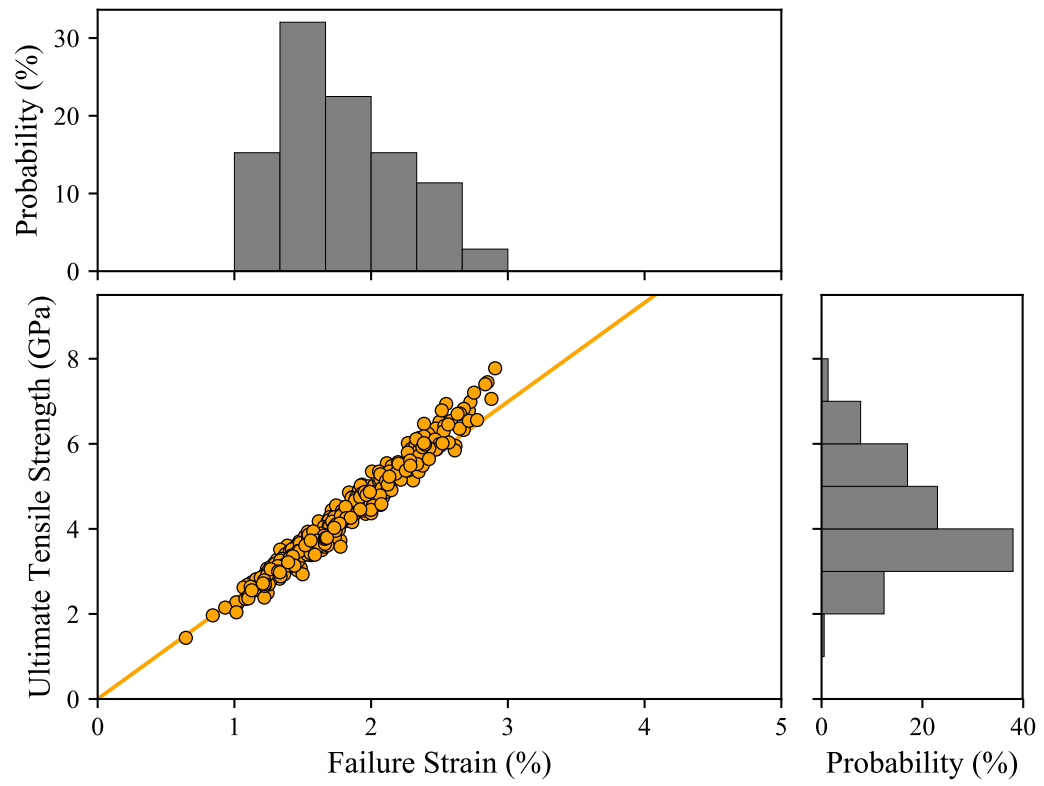

Figure 13. Fibre strength plotted against failure stress for all fibres, along with corresponding histograms, for tensile tests done at gauge lengths of $30 \mathrm{~mm}$. The straight line represents the mean Young's modulus.

If the standard 2-parameter Weibull distribution is used, there would be a deviation between the fibre strength data points and the Weibull model, more prominently in the lower region of the strength range. When a smaller data set is used for analysis, this behaviour is usually not very prominent and may go unnoticed. Since most previous studies have used between 10-50 fibre strength data points for the statistical analysis, it is very likely that any such non-linear behaviour could have been overlooked.

To understand the reasons for this deviation, it is important to understand the definition of the 2-parameter Weibull distribution. The 2-parameter Weibull distribution given by Equation 1 is obtained by starting with the generalized version given by Equation 2 and fixing the location parameter $\sigma_{u}=0$, thus making the assumption that the minimum possible fibre strength value is 0 . However, the experimentally determined data set was found to have a minimum strength value of around $2 \mathrm{GPa}$ for all gauge lengths. These data sets do not contain many values in the range 0-2 GPa. If these incomplete data sets are used for fitting to a 2-parameter Weibull distribution, the deviation from linearity in the lower regions of strength would be observed. The reason for the absence of weaker strength data points in the set may be the fibre preselection effect. Since the diameter of carbon fibres is very small, they have very low breaking forces. Many weak fibres are therefore not able to survive the applied external 


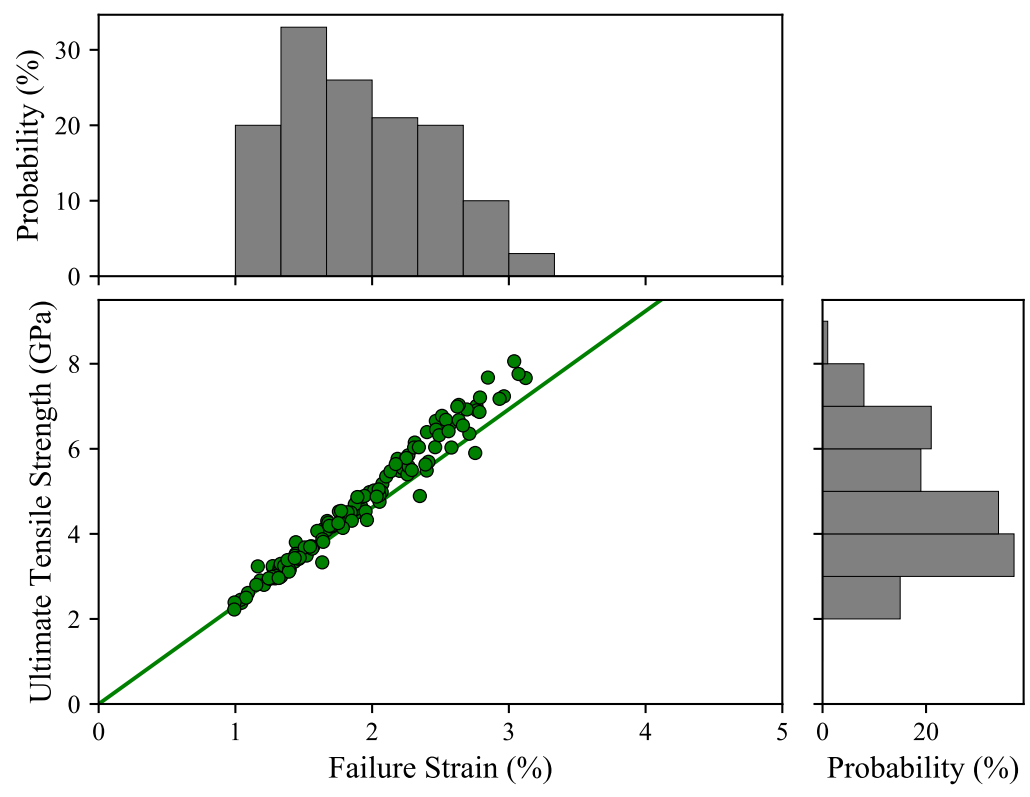

Figure 14. Fibre strength plotted against failure stress for all fibres, along with corresponding histograms, for tensile tests done at gauge lengths of $20 \mathrm{~mm}$. The straight line represents the mean Young's modulus

loads during specimen preparation and break during the process of fibre extraction and preparation. This elimination of these weak fibre strengths from the data set causes a deviation in linearity when represented on a logarithmic scale.

However, if a 3-parameter Weibull distribution (Equation 2) is used for representing the fibre strength data, it would be able to capture the non-linear behaviour of fibre strength. It may be tempting to conclude that the 3-parameter Weibull distribution is the best representative statistical function for fibre strengths as it fits the experimental data points in a better way. However, the problem does not lie necessarily with the statistical function. The experimental data itself may not be a correct representative of the actual fibre population, due to the fibre preselection effect explained earlier. Hence, using the 3-parameter Weibull distribution to represent the fibre strength behaviour may not be very appropriate either and a distribution that could better represent the fibre strengths is required. There is a need to analyse the data set statistically, to find an appropriate distribution that could represent this incomplete data set and also help in estimating a function that would be able to represent the actual fibre strength behaviour more accurately. A generalized Weibull analysis to determine the fibre strength behaviour using such experimental fibre strength data has been proposed ${ }^{54}$. 


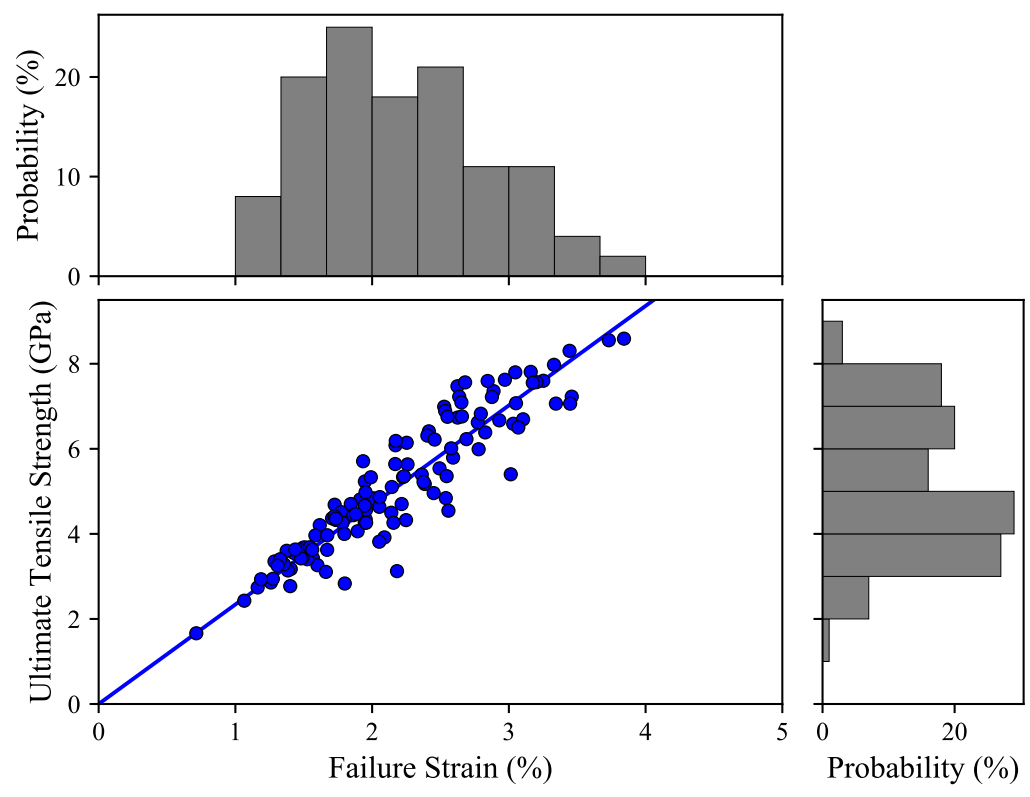

Figure 15. Fibre strength plotted against failure stress for all fibres, along with corresponding histograms, for tensile tests done at gauge lengths of $4 \mathrm{~mm}$. The straight line represents the mean Young's modulus

With this analysis a statistical function for representing the actual fibre strength variation in such brittle fibres can be determined.

\subsection{Comparison between automated and manual single fibre testing methods}

The points of similarities and differences between the automated and classical single fibre testing process have been highlighted in Table 5 .

\subsection{Advantages of the improved testing method}

(1) Alignment : ASTM C1557 recommends that the axis of the fibre should be coaxial with the line of action of the testing machine within $d$, to prevent spurious bending strains and stress concentrations ${ }^{31}$.

$$
d \leq L_{0} / 50
$$

where; $d=$ the tolerance, $(\mathrm{m})$, and $L_{0}=$ the fibre gauge length, (m).

Specimen preparation using paper tabs requires utmost precision and depends strongly on user skill to align the fibres properly on the paper tab. The paper tab 

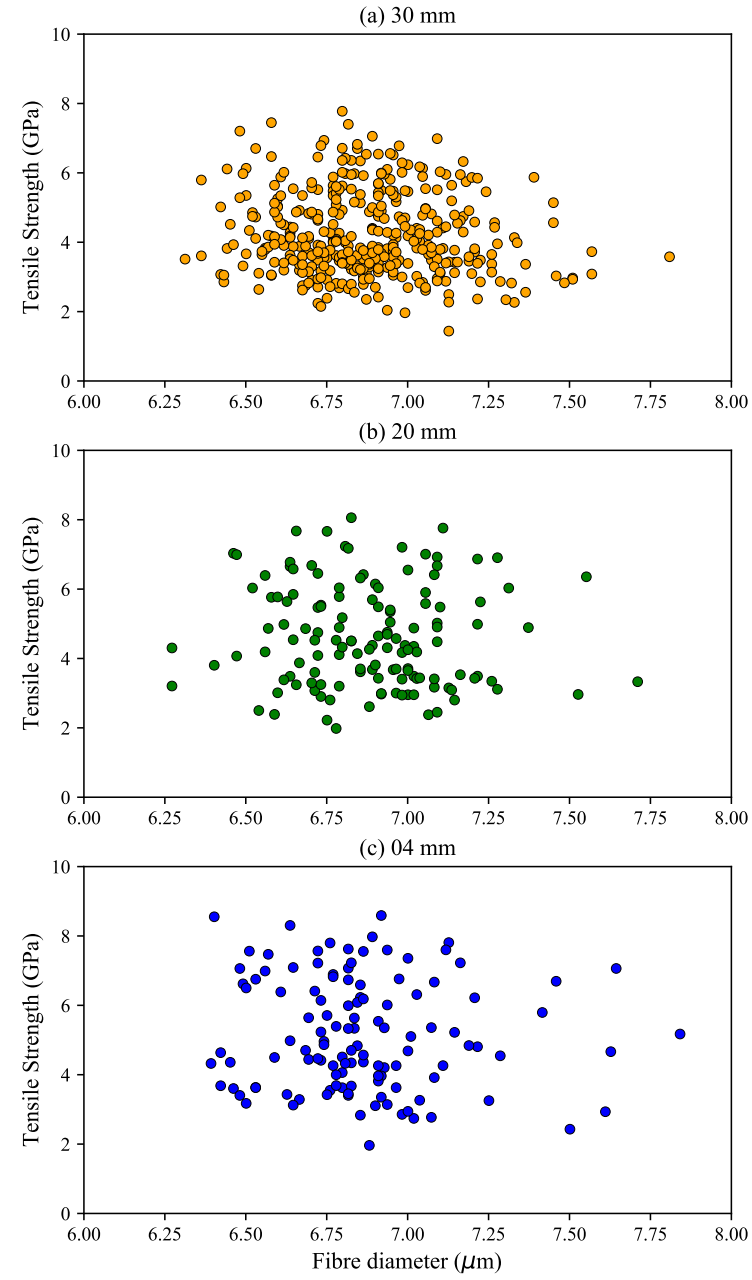

Figure 16. Fibre strength plotted against fibre diameter for all fibres, for tensile tests done at all the different gauge lengths

then has to be aligned to the loading direction to achieve proper alignment between fibre and loading direction. This makes it very difficult to achieve a proper degree of alignment while using this method, especially for smaller gauge lengths. By 


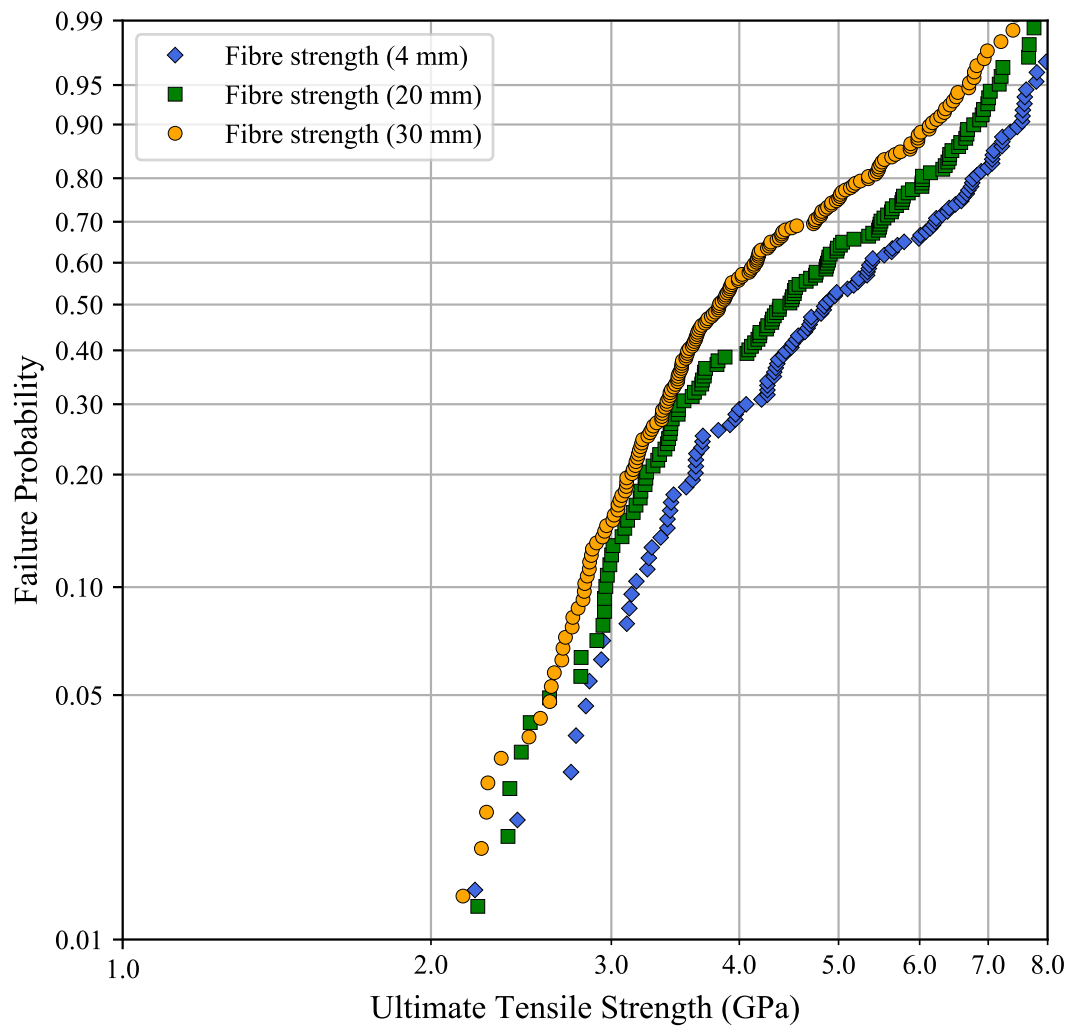

Figure 17. Fibre strength data points for all three gauge lengths represented on a logarithmic scale

comparison, in the new method grooves on plastic tabs lock any lateral movement of fibres and align the fibres properly in the direction along the length of the tab.

(2) Gauge length correction : The system has provisions for pre-tensioning the fibres to remove any slack in the fibre specimen. This may change the original separation between plastic tabs and hence the effective gauge length. The system measures the exact extension applied for tensioning and corrects the effective gauge length accordingly before proceeding with the test. This is not possible when using the manual method of paper tabs.

(3) Specimen preparation time : Using the manual method, the total time required to prepare a set of 20 fibre specimens is around 3.5 hours. This includes preparation of paper tabs of precise dimensions, mounting single fibres on each tab and the 
Table 5. Comparison of automated and manual single fibre testing methods

\begin{tabular}{|c|c|c|}
\hline & Manual testing & Automated testing \\
\hline $\begin{array}{l}\text { Specimen prepa- } \\
\text { ration }\end{array}$ & Fibre mounted on flat paper tabs & $\begin{array}{l}\text { Fibre mounted on plastic tabs } \\
\text { with grooves }\end{array}$ \\
\hline Fibre extraction & Hand held & Using vacuum/suction pen \\
\hline $\begin{array}{l}\text { Fibre mounting } \\
\text { process }\end{array}$ & Manual & Using vacuum/suction pen \\
\hline Fibre alignment & Depends upon user skill & $\begin{array}{l}\text { Near perfect alignment with the } \\
\text { help of grooves on plastic tabs }\end{array}$ \\
\hline Gauge length & $\begin{array}{l}\text { Depends on size of paper tab and } \\
\text { locations of glue (which depends } \\
\text { on the accuracy of paper tab } \\
\text { length) }\end{array}$ & $\begin{array}{l}\text { Fixed due to locking of plastic } \\
\text { tabs in cassettes }\end{array}$ \\
\hline $\begin{array}{l}\text { Specimen prepa- } \\
\text { ration time (set of } \\
20 \text { fibres) }\end{array}$ & $\begin{array}{l}\sim 3.5 \text { hours (excluding adhesive } \\
\text { curing time) } \\
\sim 12 \text { hours (including adhesive } \\
\text { curing time) }\end{array}$ & $\begin{array}{l}\sim 0.75 \text { hours (excluding adhesive } \\
\text { curing time) } \\
\sim 1 \text { hour (including adhesive cur- } \\
\text { ing time) }\end{array}$ \\
\hline $\begin{array}{l}\text { Specimen mount- } \\
\text { ing process }\end{array}$ & $\begin{array}{l}\text { Manually lifted and placed on test } \\
\text { setup }\end{array}$ & $\begin{array}{l}\text { Automated transfer of specimens } \\
\text { from cassettes to test setup using } \\
\text { Dia-Stron ALS }\end{array}$ \\
\hline $\begin{array}{l}\text { Gauge length cor- } \\
\text { rection }\end{array}$ & No & $\begin{array}{l}\text { Gauge length corrected after pre- } \\
\text { tensioning the fibres }\end{array}$ \\
\hline Intermediate Step & $\begin{array}{l}\text { Paper tabs slit manually to allow } \\
\text { load transfer }\end{array}$ & No intermediate step \\
\hline Load application & Automatic & Automatic \\
\hline $\begin{array}{l}\text { Specimen } \\
\text { time (set of } 20 \\
\text { fibres) }\end{array}$ & $\sim 1.5$ hours (Manual) & $\begin{array}{l}\sim 1.5 \text { hours (Automated, can be } \\
\text { left to run on its own) }\end{array}$ \\
\hline Total time & 5 hours & 2.5 hours \\
\hline $\begin{array}{l}\text { Total person } \\
\text { hours (set of } 20 \\
\text { fibres) }\end{array}$ & 5 hours & 1 hour \\
\hline
\end{tabular}

application of adhesive to fix the gauge length as required. The specimens are then left to cure for more than 12 hours. The individual specimens are then transferred to the test setup one at a time for experimentation. The testing process for a set of 20 specimens takes about 1.5 hours in total. Using the automated system cuts down the specimen preparation time to around 1 hour including the time required for curing. The prepared specimens can then be mounted onto a cassette following which the entire testing process is automated. The automated process reduces the total preparation and testing time to around 2.5 hours, with an effective involvement time of only about 1 hour compared to more than 5 hours for the manual process. This allows users to test a large number of fibres in a comparatively much smaller time. 
(4) Reduced specimen failure during preparation and transport : While using the manual testing process, the specimen is subjected to many external and unwanted forces at different stages of preparation and transport. This often leads to specimen failure before the actual test. Using an automated system eliminates most unwanted and accidental forces which help in preserving the majority of specimens for the final test.

(5) Ability to test at shorter gauge lengths : Using a cassette-based specimen set simplifies the preparation process for single fibre specimens and the added grooves on plastic tabs ensure proper alignment of fibres. This allows specimens to be prepared and testted at a reduced gauge length. The present system thus allows fibre specimens to be prepared and tested at gauge length as small as $4 \mathrm{~mm}$. However, this requires extra care to minimise any clamping effect.

\subsection{Scope for improvement}

(1) Gauge length : Practical problems are encountered when testing fibres at either very small or very large gauge lengths. The effect of clamping and misalignment would be significantly greater in the case of fibre specimens with a very small gauge length. Conversely, testing fibres at very large gauge lengths may eliminate weaker fibres which may break prematurely during handling, and the effect of preselection would be greater. From a practical point of view, there may therefore be an optimal gauge length that would minimize the combined effect of clamping and fibre preselection. It would be useful to determine this gauge length and use it as a standard.

(2) Clamping effects/Determination of failure location : A valid test result is considered to be one in which fibre failure does not occur in the gripping region. However, using either the manual or automated methods it is difficult to locate the exact region of break after failure occurs. Determining the exact region of failure would help in eliminating improper test results.

(3) Diameter variation : To determine the fibre strength, the measured failure load is divided by the fibre cross-sectional area. Due to the very small fibre size, measuring the fibre cross-section can be very difficult. The usual practice is to measure the fibre diameter at one particular location along the gauge length of the fibre and then calculate the area based on the assumption of circularity in fibre section. Depending upon the type and manufacturing process used, this may not always be an accurate assumption and there may be variations in diameter along the axial or angular direction, as already shown in section 4.1. For determining the accurate fibre strength value, the cross-sectional area at the exact location of failure would be required. This point further highlights the need to determine the exact failure location of the fibre.

\section{Conclusions}

Tensile strength and dimensional variation of T700 carbon fibres, a popularly used reinforcement for composite materials in critical structural applications, have been 
investigated. An attempt has been made to understand the critical issues which limit the accuracy of experimentally generated fibre strength results. Different experimental challenges in fibre strength characterisation associated primarily with single fibre tests are discussed. Improvements in the single fibre testing process have been made by overcoming the existing experimental limitations. Automating the testing process has been shown to facilitate the generation of a large set of required experimental data for a more detailed statistical analysis.

The improved testing methodology has been used to generate fibre strength data for the T700 carbon fibres at three different gauge lengths: 4, 20 and $30 \mathrm{~mm}$. The data set used for analysis is much larger than the ones used for any other studies reported in literature. Fibre strength has been shown to be scattered in a range of 2-8 GPa with shorter fibres having larger strengths on average, in agreement with the weakest link theory. Although the tests at different gauge lengths yielded very similar Young's moduli, it was found that the coefficient of variation was very large for tests conducted at shorter gauge lengths. This is because the testing of short fibres is associated with many experimental issues which restrict the accuracy of the measurements made. For many composite strength models, fibre strength information at very short gauge lengths is required. However, it has been shown that conducting tensile tests at very short gauge lengths can lead to inaccurate results.

Preliminary statistical analysis of the generated fibre strength data has shown that the standard Weibull distributions may not be appropriate to accurately capture the intrinsic fibre strength variation; and a misfit between the experimental fibre strength and the Weibull model is observed. It is very likely that this behaviour may have been overlooked by the previous studies as the sample sizes used for those cases were comparatively much smaller.

A detailed dimensional analysis of the fibre surface was also conducted. It was found that not all fibres have a circular cross-section, which is contrary to the popular assumption. Existence of a non-circular fibre cross-section limits the use of the existing methods of fibre strength determination and statistical analysis.

\section{Acknowledgements}

The research leading to these results has been done within the framework of the FiBreMoD project and has received funding from the European Union's Horizon 2020 research and innovation programme under the Marie Sklodowska-Curie grant agreement No 722626. The authors would also like to thank Chomarat Ltd. for providing the T700 fibre bobbins for the study.

\section{References}

1. Thionnet A, Chou HY, Bunsell A. Fibre Break Failure Processes in Unidirectional Composites. Part 2: Failure and Critical Damage State Induced by Sustained Tensile Loading. Applied Composite Materials. 2015 Apr;22(2):141-155.

2. Blassiau S, Thionnet A, Bunsell AR. Micromechanisms of load transfer in a unidirectional carbon fibre-reinforced epoxy composite due to fibre failures. Part 1: Micromechanisms and 3D analysis of load transfer: The elastic case. Composite Structures. 2006;74(3):303-318. 
3. Swolfs Y, Verpoest I, Gorbatikh L. Issues in strength models for unidirectional fibrereinforced composites related to Weibull distributions, fibre packings and boundary effects. Composites Science and Technology. 2015;114:42 - 49.

4. Bunsell AR, Thionnet A. Life prediction for carbon fibre filament wound composite structures. Philosophical Magazine. 2010;90(31-32):4129-4146.

5. Islam F, Joannès S, Laiarinandrasana L. Evaluation of Critical Parameters in Tensile Strength Measurement of Single Fibres. Journal of Composites Science. 2019 Sep;3(3):69.

6. Islam F, Joannès S, Bucknell S, Leray Y, Bunsell A, Laiarinandrasana L. Improvements in determination of carbon fibre strength distribution using automation and statistical data analysis. In: Proceedings of the Fiber Society Spring 2018 Conference; June, 2018. .

7. Thomason JL. On the application of Weibull analysis to experimentally determined single fibre strength distributions. Composites Science and Technology. 2013;77:74 - 80.

8. Berger MH, Jeulin D. Statistical analysis of the failure stresses of ceramic fibres: Dependence of the Weibull parameters on the gauge length, diameter variation and fluctuation of defect density. Journal of Materials Science. 2003 Jul;38(13):2913-2923.

9. Bunsell AR, Joannès S, Marcellan A. Handbook of Properties of Textile and Technical Fibres. Woodhead Publishing; 2018.

10. Swolfs Y, Verpoest I, Gorbatikh L. A review of input data and modelling assumptions in longitudinal strength models for unidirectional fibre-reinforced composites. Composite Structures. 2016;150:153 - 172 .

11. Weibull W. A Statistical Distribution Function of Wide Applicability. Journal of Applied Mechanics. 1951:293-297.

12. Andersons J, Joffe R, Hojo M, Ochiai S. Glass fibre strength distribution determined by common experimental methods. Composites Science and Technology. 2002;62(1):131 145.

13. Bensadoun F, Verpoest I, Baets J, Müssig J, Graupner N, Davies P, et al. Impregnated fibre bundle test for natural fibres used in composites. Journal of Reinforced Plastics and Composites. 2017;36(13):942-957.

14. Creasy TS. A method of extracting Weibull survival model parameters from filament bundle load/strain data. Composites Science and Technology. 2000;60(6):825 - 832.

15. Phoenix SL. Probabilistic strength analysis of fibre bundle structures. Fibre Science and Technology. 1974;7(1):15 - 31 .

16. Abramchuk SS, Ermolenko AF, V D P. Estimating the characteristics of reinforcing fibers by testing fiber bundles. Mechanics of Composite Materials. 1984;20(1):1 - 5.

17. Hill R, Okoroafor EU. Weibull statistics of fibre bundle failure using mechanical and acoustic emission testing: the influence of interfibre friction. Composites. 1995;26(10):699 -705 .

18. Ermolenko AF, Abramchuk SS, Protasov VD. Evaluating parameters of the strength distribution of reinforcing fibers interacting on a lateral surface by testing their bundles. Mechanics of Composite Materials. 1985 Jan;21(1):1-4.

19. Yavin B, Gallis HE, Scherf J, Eitan A, Wagner HD. Continuous monitoring of the fragmentation phenomenon in single fiber composite materials. Polymer Composites. 1991;12(6):436-446.

20. Shioya M, Takaku A. Estimation of fibre and interfacial shear strength by using a singlefibre composite. Composites Science and Technology. 1995;55(1):33 - 39. 
21. Andersons J, Joffe R, Hojo M, Ochiai S. Fibre fragment distribution in a single-fibre composite tension test. Composites Part B: Engineering. 2001;32(4):323 - 332.

22. Hui CY, Shia D, Berglund LA. Estimation of interfacial shear strength: an application of a new statistical theory for single fiber composite test. Composites Science and Technology. 1999;59(13):2037 - 2046.

23. Zhao FM, Okabe T, Takeda N. The estimation of statistical fiber strength by fragmentation tests of single-fiber composites. Composites Science and Technology. 2000;60(10):19651974.

24. Thuvander F, Gamstedt EK, Ahlgren P. Distribution of strain to failure of single wood pulp fibres. Nordic Pulp and Paper Research Journal. 2001;16(1):46-56. Cited By 14.

25. Detassis M, Pegoretti A, Migliaresi C, Wagner HD. Experimental evaluation of residual stresses in single fibre composites by means of the fragmentation test. Journal of Materials Science. 1996 May;31(9):2385-2392.

26. Tsai SW, Hahn HT. Introduction to composite materials. Technomic; 1980.

27. Curtin WA. Exact theory of fibre fragmentation in a single-filament composite. Journal of Materials Science. 1991;26(19):5239-5253. Cited By 194.

28. Andersons J, Joffe R, Sandmark R. Constrained fragmentation of composites under uniaxial loading. Mechanics of Composite Materials. 1995;31(1):26-33. Cited By 12.

29. Hui CY, Phoenix SL, Ibnabdeljalil M, Smith RL. An exact closed form solution for fragmentation of Weibull fibers in a single filament composite with applications to fiberreinforced ceramics. Journal of the Mechanics and Physics of Solids. 1995;43(10):15511585. Cited By 101.

30. Curtin WA. Fiber fragmentation in a single-filament composite. Applied Physics Letters. 1991;58(11):1155-1157.

31. ASTM C1557 - 14 Standard Test Method for Tensile Strength and Youngs Modulus of Fibers;

32. ISO 11566:1996 Carbon fibre - Determination of the tensile properties of single-filament specimens;

33. ASTM D3379-75 Standard Test Method for Tensile Strength and Young's Modulus for High-Modulus Single-Filament Materials;

34. Kelly A, Tyson WR. Tensile properties of fibre-reinforced metals - copper/tungsten and copper/molybdenum. J Mech Phys Sol. 1965;13(329).

35. Phoenix SL, Sexsmith RG. CLAMP EFFECTS IN FIBER TESTING. Journal of Composite Materials. 1972;6:322-337.

36. Stoner EG, Edie DD, Durham SD. An end-effect model for the single-filament tensile test. Journal of Materials Science. 1994;29(24):6561-6574.

37. Gulino R, Phoenix SL. Weibull strength statistics for graphite fibres measured from the break progression in a model graphite/glass/epoxy microcomposite. Journal of Materials Science. 1991;26(11):3107-3118.

38. Phoenix SL, Schwartz P, Robinson HH. Statistics for the strength and lifetime in creep-rupture of model carbon/epoxy composites. Composites Science and Technology. 1988;32(2):81-120.

39. Yu W, Yao J. Tensile strength and its variation of PAN-based carbon fibers. I. Statistical distribution and volume dependence. Journal of Applied Polymer Science. 2006;101(5):3175-3182. 
40. Goda K, Fukunaga H. The evaluation of the strength distribution of silicon carbide and alumina fibres by a multi-modal Weibull distribution. Journal of Materials Science. 1986;21(12):4475-4480.

41. Beyerlein IJ, Phoenix SL. Statistics for the strength and size effects of microcomposites with four carbon fibers in epoxy resin. Composites Science and Technology. 1996;56(1):75-92.

42. Yang L, Thomason JL. Effect of silane coupling agent on mechanical performance of glass fibre. Journal of Materials Science. 2013;48(5):1947-1954.

43. Yao J, Yu W. Tensile strength and its variation for PAN-based carbon fibers. II. Calibration of the variation from testing. Journal of Applied Polymer Science. 2007;104(4):2625-2632.

44. Watson AS, Smith RL. An examination of statistical theories for fibrous materials in the light of experimental data. Journal of Materials Science. 1985;20(9):3260-3270.

45. Tanaka T, Nakayama H, Sakaida A, Horikawa N. Estimation of tensile strength distribution for carbon fiber with diameter variation along fiber. Materials Science Research International. 1999;5(2):90-97.

46. Curtin WA. Tensile strength of fiber-reinforced composites: III. Beyond the traditional Weibull model for fiber strengths. Journal of Composite Materials. 2000;34(15):1301-1332.

47. Parthasarathy TA. Extraction of Weibull Parameters of Fiber Strength from Means and Standard Deviations of Failure Loads and Fiber Diameters. Journal of the American Ceramic Society. 2001;84(3):588-592.

48. Yao J, Yu W, Pan D. Tensile strength and its variation of PAN-based carbon fibers. III. Weak-link analysis. Journal of Applied Polymer Science. 2008;110(6):3778-3784.

49. Garat W, Corn S, Moigne NL, Beaugrand J, Bergeret A. Dimensional variations and mechanical behaviour of natural fibres from various plant species in controlled hygro/hydrothermal conditions. In: Proceedings of the 18th European Conference on Composite Materials (ECCM-2018); June, 2018. .

50. Dia-Stron website; accessed April 22, 2019. Available from: http://www. diastron. com.

51. Thionnet A, Chou HY, Bunsell A. Fibre Break Failure Processes in Unidirectional Composites. Part 1: Failure and Critical Damage State Induced by Increasing Tensile Loading. Applied Composite Materials. 2015 Apr;22(2):119-140.

52. Curtis WA, Milne JM, Reynolds WN. Non-Hookean Behaviour of Strong Carbon Fibres. Nature. 1968;220:1024-1025.

53. Joannès S, Islam F, Laiarinandrasana L. Uncertainty in Fibre Strength Characterisation Due to Uncertainty in Measurement and Sampling Randomness. Applied Composite Materials. 2020 Jun;27(3):165-184.

54. Islam F, Joannès S, Bucknell S, Leray Y, Bunsell A, Laiarinandrasana L. Towards accurate and efficient single fibre characterization to better assess failure strength distribution. In: Proceedings of the 18th European Conference on Composite Materials (ECCM-2018); June, 2018. . 